\title{
Electronic Patient Visit Assessment
}

National Cancer Institute

\section{Source}

National Cancer Institute. Electronic Patient Visit Assessment. NCI Thesaurus. Code C154266.

A web-based instrument that is used capture a patient's self-assessments prior to a healthcare encounter. 